Meta

Journal des tradlucteurs

Translators' Journal

\title{
Que la lumière soit !... sur les conducteurs, fils, câbles et cordons
}

\section{Marie-Lourdes Valcin}

Volume 23, numéro 2, juin 1978

URI : https://id.erudit.org/iderudit/004518ar

DOI : https://doi.org/10.7202/004518ar

Aller au sommaire du numéro

Éditeur(s)

Les Presses de l'Université de Montréal

ISSN

0026-0452 (imprimé)

1492-1421 (numérique)

Découvrir la revue

Citer cet article

Valcin, M.-L. (1978). Que la lumière soit !... sur les conducteurs, fils, câbles et cordons. Meta, 23(2), 159-161. https://doi.org/10.7202/004518ar d'utilisation que vous pouvez consulter en ligne.

https://apropos.erudit.org/fr/usagers/politique-dutilisation/ 


\section{QUE LA LUMIÈRE SOIT !... sur les conducteurs, fils, câbles et cordons}

Voici quatre mots dont tout ce que nous savons au départ est qu'ils appartiennent au vocabulaire de l'électronique et qu'il y a un certain rapport de synonymie entre eux. Il s'agit maintenant de définir ce rapport d'une façon précise et de mettre en lumière, le cas échéant, la spécificité de chacun des termes.

La consultation des dictionnaires généraux nous apprend que le fil, le câble et le cordon servent tous à transporter le courant électrique. Nous pourrions donc nous croire autorisés à considérer le mot «conducteur » comme le «père » de la famille synonymique des fils, câbles et cordons. Mais les choses ne sont pas si simples. Dans le Grand Larousse Encyclopédique ${ }^{1}$, nous retrouvons l'expression «câbles à conducteurs multiples» qui indique que le conducteur peut être un élément du câble. Le GLE définit le conducteur comme un fil métallique servant à conduire l'énergie électrique : «Pour de faibles intensités, on emploie des fils, c'est-à-dire des conducteurs, simples ${ }^{2}$. " Paradoxalement, nous relevons, quelques paragraphes plus loin, le passage suivant : « La technique du transport à haute / fréquence nécessite l'emploi de conducteurs spéciaux, dits câbles coaxiaux, comportant un fil central en cuivre ${ }^{3}$. $\$$ Et voilà que notre belle synonymie conducteur-fil s'envole en fumée, puisque le fil n'est ici qu'un composant du conducteur.

Voyons maintenant le point de vue des spécialistes. La revue Équipement électrique et électronique, pour ne citer qu'un exemple, parle de «conducteurs aluminium-acier dans lesquels une âme centrale en fils d'acier / est entourée de fils d'aluminium ${ }^{4} »$. Ceci nous ramène à notre conception première du conducteur comme terme générique désignant l'ensemble du matériel qui sert à trainsporter l'énergie électrique.

Il nous reste à analyser les relations existant entre les trois membres de la famille des conducteurs, soit le fil, le câble et le cordon.

L'Encyclopédie internationale des sciences et des techniques définit les fils électriques comme «des cylindres ou des filaments métalliques ${ }^{5} 》$. Cette description correspond parfaitement à celle de l'équivalent anglais de fil : "Wire : a line of wire for conducting electrical current ${ }^{6} . \gg \mathrm{Du}$ strict point de vue technique, le fil n'est donc que l'élément de base à partir duquel on fabrique les divers conducteurs. Mais la langue courante s'est permis quelques accrocs à cette définition technique, et, dans la pratique, le mot fil sert à désigner plusieurs

1. Grand Larousse Encyclopédique, Paris, Larousse, 1960, vol. 2, entrée : câble.

2. Larousse, op. cit., vol. 4, entrée : fil.

3. Larousse, op. cit., vol. 4, entrée: fil.

4. « Cuivre ou aluminium», in Equipement électrique et électronique, Paris, mars 1977 , p. 37.

5. Encyclopédie internationale des sciences et des techniques, Paris, Presses de la Cité, 1970 , vol. 2 , p. 549, entrée : câble électrique.

6. Webster's Third New International Dictionary, Springfield (Mass.), Merriam Company, 1971, p. 2623. 
conducteurs qui sont en réalité des câbles ou des cordons. $\AA$ preuve cette observation du GLE : «Les câbles sans armature extérieure servent couramment sous le nom de fils électriques ${ }^{7}$.

C'est au niveau de la comparaison entre câble et cordon que les choses se corsent un peu. On pourrait utiliser plusieurs critères de différenciation. Il y a tout d'abord les caractéristiques physiques. Le câble électrique est « un conducteur / de grande longueur ${ }^{8}$ ». «Le plus long câble en service est celui qui relie l'île Vancouver / à Southport (Australie); il mesure $14516 \mathrm{~km}^{9}$. 》 En revanche, le cordon est généralement ou relativement court ; exemple : «cordon amovible de $1,50 \mathrm{~m}^{10} \gg$. Cette caractéristique tient à l'usage auquel le cordon est destiné, usage que nous verrons plus loin. La section est un autre critère de comparaison. Pour les câbles, la section peut atteindre 1200 millimètres carrés ${ }^{11}$, alors que pour les cordons elle est très faible. De plus, le câble peut être « rigide, semi-rigide, flexible ou extra-flexible ${ }^{12} »$, tandis que le cordon est toujours « très souple ${ }^{13}$ ».

Autre facteur de différenciation : les éléments constitutifs. Un câble peut être constitué de "quelques milliers de fils élémentaires tressés entre eux ${ }^{14}$ ». Pour le cordon, le nombre de fils est généralement de deux, comme l'atteste cette définition de «cord »: « a small flexible / cable usu. consisting of a pair of / wires ${ }^{15}$. 》 De plus, le câble peut être nu ou isolé, alors que le cordon est toujours isolé ; là encore, cela tient à l'usage auquel le cordon est destiné. Enfin, contrairement au câble, le cordon comporte toujours une fiche à l'une de ses extrémités.

Dernier critère de comparaison : la destination. Le câble est un conducteur tout-usage ; il peut servir pour l'éclairage, les télécommunications, l'informatique, le soudage, etc. En revanche, le champ d'utilisation du cordon est beaucoup plus restreint : il s'agit en fait presque exclusivement du domaine des petits et moyens appareils électroménagers, comme l'indiquent les définitions suivantes : "conducteur / servant surtout à alimenter les appareils d'usage domestique ${ }^{16} \gg$; «cable / used to connect a lamp, electric iron / or other appliance with a receptacle ${ }^{17}$. $\gg \mathrm{Il}$ est intéressant de noter que les gros appareils électroménagers utilisent un câble plutôt qu'un cordon, comme en témoigne la mention suivante relevée dans une revue spécialisée : «câbles durables pour les fours électriques ${ }^{18}{ }^{»}$. C'est la destination du cordon qui explique qu'il soit toujours court, isolé et flexible.

7. Larousse, op. cit., vol. 2, entrée : câble.

8. Presses de la Cité, op. cit., vol. 2 , p. 549, entrée : câble électrique.

9. Larousse, op. cit., vol. 2 , entrée : câble.

10. L'électricien, Paris; Dunod, no 2148 , mars 1973, p. 11 (description du chauffe-plats).

11. «Cuivre ou aluminium », ibid., p. 37 .

12. Presses de la Cité, op. cit., vol. 2, p. 549 , entrée : câble électrique.

13. Larousse, op. cit., vol. 3 , entrée : cordon.

14. Presses de la Citê, op. cit., vol. 2, p. 549, entrée : câble électrique.

15. Webster, op. cit., p. 505.

16. Larousse, op. cit., vol. 3 , entrée : cordon

17. Webster, op. cit., p. 505 .

18. * Fiches techniques $\gg$, in L'électricien, $n^{\circ} 2135$, janvier 1972, p. 21. 
Par ailleurs, le câble sert à transporter des courants de forte intensité ${ }^{10}$ et le cordon, des courants beaucoup plus faibles. C'est ce qui explique qu'une lampe utilise un cordon et un four électrique, un câble.

En résumé :

Conducteur sert à désigner tout ce qui transporte l'énergie électrique (anglais : conductor).

Fil désigne l'élément de base de tout conducteur, mais aussi, en langue courante, des câbles et cordons (anglais : wire).

Câble désigne un conducteur long, isolé ou non, servant à de multiples usages (surtout industriels) et transportant des courants de forte intensité (anglais : cable).

Cordon désigne un conducteur court, isolé, servant à alimenter les appareils d'usage domestique, et transportant des courants de faible intensité (anglais : cord).

... ET LA LUMIÈRE FUT

(du moins nous l'espérons).

Marie-Lourdes VAlcin 